

R. J. Howerton

Manuscript Date: May 10, 1983

LAWRENCE LIVERMORE LABORATORY University of California $\bullet$ Livermore, California $\bullet 94550$ 


\section{Fenewerd}

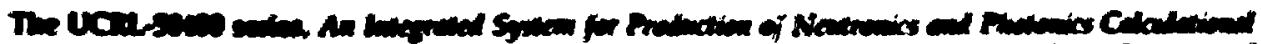

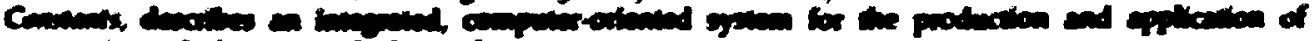

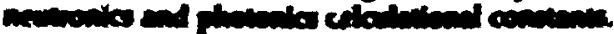

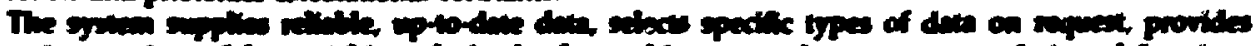

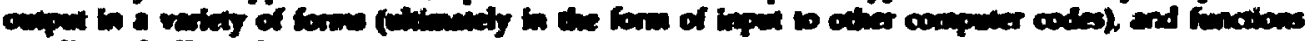
rividy and endienty.

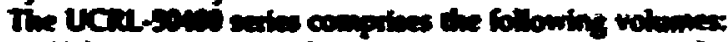

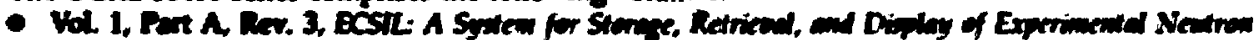
Dera, Sepleanber 1975.

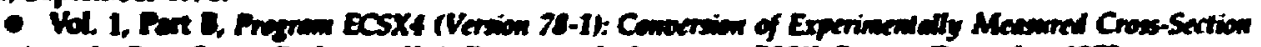





- Vol. 3, Rev. 2, An Imiex of the Expertimentel Data of Nentron-Indaced Imeeractions, July 1976.

- Vol. 1, Rev. 1, Exuladed Nucleer Duta Litrary. Sepiember 1901.

- Vol.5, Part A, Rev. 1, CLYDE: A Cade for the Production of Calculational Constanls from Ni lear Data. September 1975.

- Vol. 5, Part B, Rev. 1, Relativistic Transformations between Center-of-Mass and Laboratory Systems for Trmo-Body Nuclear Reactions, April 1978.

- Vol. 6, Rev. 2, Tables and Graphs of Photom-Interaction Cross Sections from 1 keV to $100 \mathrm{MeV}$. Jecember 1978 .

- Vol. 7, Part A, Rev. 1, Major Neutron-Induced Interactions ( $Z \leq 55$ ): Graphical, Experimental Data, July 1976.

- Vol. 7. Part B. Rev. 1, Major Neutron-Induced Interactions ( $Z>$ 55): Graphical, Experimental Data, July 1976.

- Vol. 8, Part A, Rev. 1, Supplemental Neutron-Induced Interactions ( $Z \leq 35)$ : Graphical, Experimemtal Data. July 1976.

- Vol. 8, Part B, Rev. 1, Supplemental Neutron-Induced Interactions (Z > 35): Graphical, Experimermal Data, July 1976.

- Vol. 9. Thresholds of Nuclear Reactions Induced by Neutrons, Photons, Deuterons, Tritons, and Alpha Particles, September 1970.

- Vol. 10, Rev. 1, Tabulated Experimental Data for Neutron-Induced Interactions, July 1976.

- Vol. 11, Experimental Data, Indexes, and Techniques of Obtainirg a Selected Set of Neutron Resonance Parameters, May 1972.

- Vol. 12, An Atlas of Resolved Neulron Resonance Parameters, July 1972.

- Vol. 13, An Atlas of Unresolved Neutron Resonance Parameters, September 1972.

- Vol. 14, TARTNP: A Coupled Neutron-Phuton Monte Carlotransport Code, February 1976.

- Vol. 15, Part A, The LLL Evaluated-Nuclear-Data Library (ENDL): Evaluation Techniques, Reaction Index, and Descriptions of Individual Evaluations, September 1975.

- Vol. 15, Part B, Rev. 1, The LLL Evaluated-Nuclear-Data Library (ENDL): Graphs of Cross Sections from the Library, October 1978.

- Vol. 15, Part C, The LLL Evaluated-Nuclear-Data Library (ENDL): Transiation of ENDL NeutronInduced Interaction Data into the ENDF/B Format, April 1976.

- Vol. 15, Part D, Rev. 1, The LLL Evaluated-Nuclear-Data Library (ENDL): Descriptions of Individual Evnluations for $Z=0-98$, May 1978.

- Vol. 15, Part E, Data Testing Results for the LLL Nuclear Data Library (ENDL-78), August 1979.

- Voi. 15, Part F, Experimental and Evaluated Elastic Nuclear Plus Interference Cross Sections for Light Charged Particles, July 1980.

- Vol. 16, Rev. 2, Tabular and Graphical Presentation of 175 Neutron-Group Constants Derived from the LLL Evaluated-Nuclear-Data Library (ENDL), October 1978.

- Vol. 17, Part A, Rev. 2, Program LINEAR (Version 79-1): Linearize Data in the Evaluated-Nuclear-Data File/Version B (ENDF/B) Format, October 1979.

- Vol. 17, Part B, Rev. 2, Program SIGMA1 (Version 79-1): Doppler Broaden Evaluated Cross Sections in the Evaluated-Nuciear-Data File/Version B (ENDF/B) Format, October 1979. 


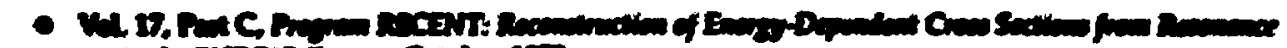

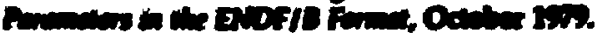

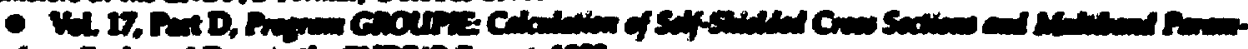



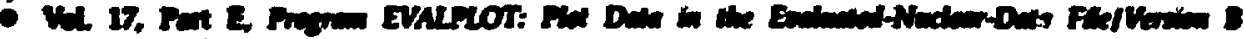


19s.



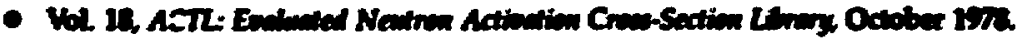

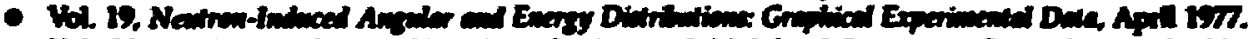

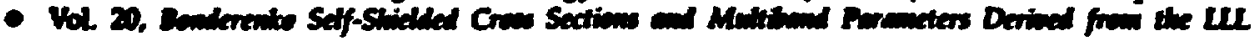



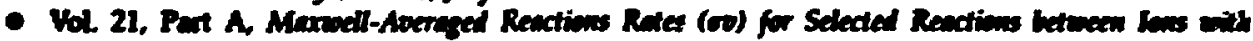
Aromic Mas $\leq 11$, February 1979.

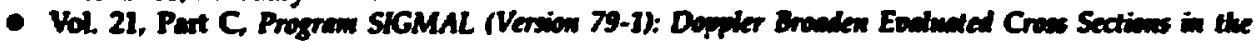
Libermore-Exolumed Nwelesr Duta Librery (ENDL) Format, March 1979.

- Vol. 22, Rev. 1, GAMIDEN: A Program to Ail in fine Identification of Unkmown Materials by GamimiRay Spectroscopy, June 1902.

- Vol. 22, Rev. 2, GAMIDEN: A Program to Aid in the Jdentification of Unknown Materials by GamimaRey Spectroscopy, May 1983.

- Vol. 23, ENSL and CDRL- Eoduated Nuclear Structure Litrries, February 1981.

- Vol. 24. Threshoids ard Q Values of Nuclear Reactions Induced by Neutrons, Protons, Deuterons. Tritons, ${ }^{3}$ He lons. Alpha Particles, and Photons, March 1981. 


\section{Comeres}

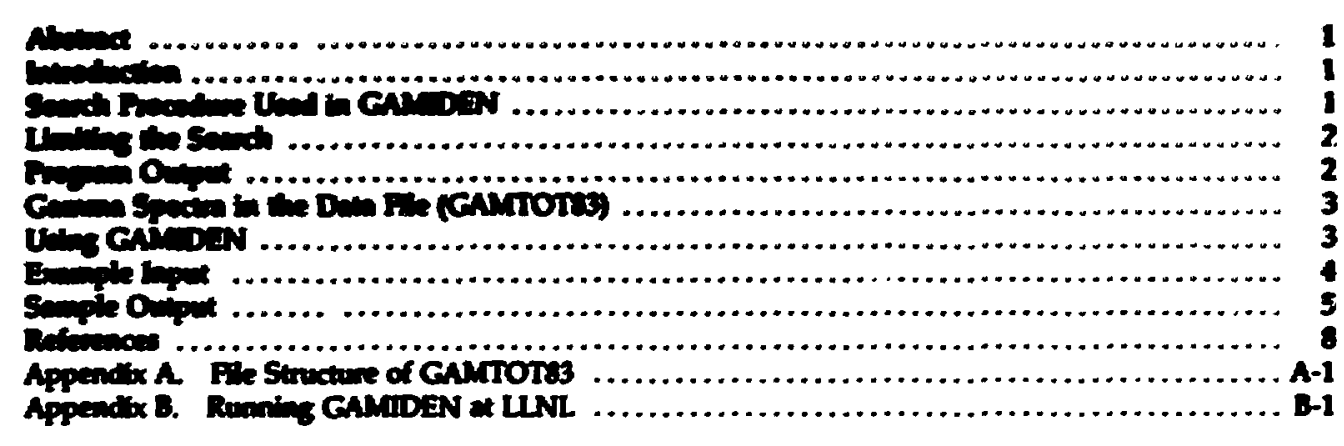




\title{
GAMDER: A Program to Aid in the Identification of Unknown Materials by Gamma-Ray Spectroscopy
}

\begin{abstract}
Abetract
The intent of the computer code GAMIDEN is to help identify isotopes by their gamma-ray emissions and thus to assist in the nondestructive assay of unknown materials. From both radioactive decays and neutron captures, GAMIIDEN searches GAMTOT83, a file of gamma-ray spectra, for matches with observed photon eniergies. This report describes the search procedure, outlines the use of the code, and gives an example.

The code is designed to operate on the CRAY 1 computer at Lawrence Livermore National Laboratory (LLNL). It is written in standard Fortran (ANSI) for the most part but contains some LRLTRAN instructions to make use of the Livermore time-sharing system (LTSS). The code uses about 545,000 words of memory. Typical problems run in about $45 \mathrm{~s}$. The source program and the data file are available on request.
\end{abstract}

\section{Introduction}

The gamma rays emitted by an isotope undergoing radioactive decay or neutron capture have energies and intensities that are characteristic of that isotope. Such a set of gamma rays is often referred to as the signature of the isotope. A useful method for the nondestructive assay of unknown materials is based on identifying the signature of the isotope.

Since there are thousands of known gamma rays, we have developed an automated search procedure. GAMIDEN, a computer code, searches GAMTOT83, a disk file of gamma-ray spectra, for isotopes that match observed gamma energies. GAMIDEN lists the matching isotopes, eliminates isotopes that are not likely to be the source of the observed photons, and then lists the isotopes that are left-the likely candidates. The user makes any further analysis required to identify the actual source isotopes.

\section{Search Procedure Used in GAMIDEN}

The search procedure used in GAMIDEN is outlined as follows:

1. Given a set of observed photon energies, search the tabulated data for photons with the same energies, within experimental uncertainty. Isotopes that emit such photons form the initial list of possible sources of the observed photons.

2. Examine the tabulated spectrum of each isotope for gammas that have a higher probability of emission than the observed gamma has; presumably, such gammas should have been observed as well.

3. Examine energies of the other observed photons to see if these higher-probability gammas, if any, were, in fact, observed.

4. If the matching isotope's spectrum contains a higher-probability gamma and it was not observed, remove this isotope from the list of possible sources. Isotopes that survive this test constitute the final list of possible sources. 


\section{Limining the Samch}

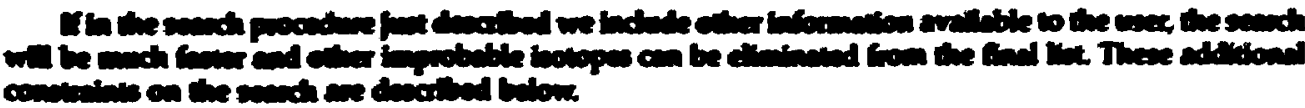

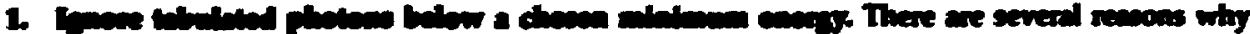

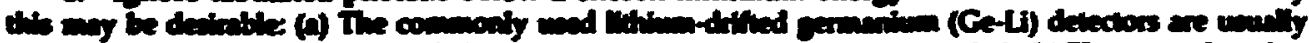

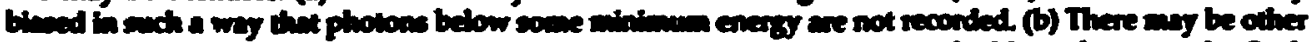



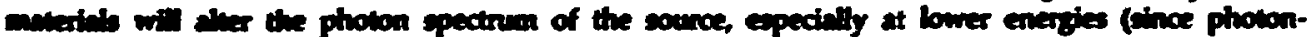


observed photons, and the user may wank to linit the volume of output from the program. He con do this by chooving an energy avoff that lenves a resoonuble number of higher-enesy photons as input vilues. If there are inderposed manerisk, higher-energy photons are most likely so represent the spectrum of the unknown source.

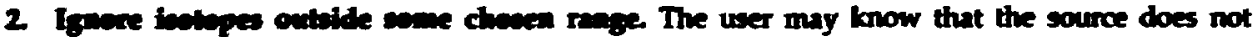
contain isotopes outside some range of atonic number $Z$ and mass number $A$, or he may simply be uninterested in inotopes outside of some such range. The user combines the $Z$ and $A$ of these limiting isotopes into numbers equal to $1000 Z+A$, and enters these to indicate the limits chosen.

3. Seanch only prempt gammo, or only those from radioactive decays, or both. If the source is not emitting prompt gammas or gammas from decays with very short half-lives, the user can specify a minimum half-life as a criterion for searching. He can set this value to zero if he wants to search both prompt and decay gammas. (In GAMTOT83 prompt gammas are assigned a half-life of $10^{-14} \mathrm{~s}$.)

(Of course, the capture process may result in the formation of a radioactive product, which will decay with its own characteristic spectrum and half-life. The user may need to consider this in deciding whether to search prompt gammas only.)

4. Do not consider gammas with a low probability of emission. Clearly low-probability photons, even if present in the unknown spectrum, may not be detected. (The tabulated probabilities, aiso called multiplicities, are actually probabilities of emission per decay or per neutron capture.)

The user will also have to estimate the uncertainty to be associated with each observed photon energy. The Ge-Li detector has an accuracy of plus or minus a few keV for photon energies in the range from $100 \mathrm{keV}$ to $10 \mathrm{MeV}$. This uncertainty arises mainly from the nonlinearity of the relationship between photon energy and detector output. (A Ge-Li detector operated at liquid-nitrogen temperature has a precision of about $0.1 \mathrm{keV}$ for photons in this energy range.) Unknown energies are determined by interpolating or extrapolating detector output values from known photons; typically, only a few such calibration photons are used. To take account of asymmetry in the calibration, the program permits the user to specify different values for the uncertainties above and below the observed energies.

\section{Program Output}

GAMIDEN provides four kinds of output information:

1. The input data (search constraints and photon energies).

2. A list of each match of an observed photon giving the identity of the matching isotope and a list of all the photons in the spectrum of the matching isotope that satisfy the search constraints.

3. A list of the isotopes that have no unobserved higher-probability photons. These isotopes remain active candidates.

4. A list of the eliminated isotopes, with the multiplicity and energy of both the observed and the unobserved higher-multiplicity photon. 


\title{
Gaman Spectra in the Duta File (GAMTromes
}

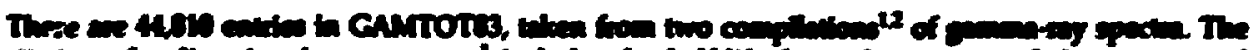

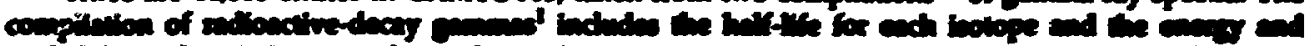

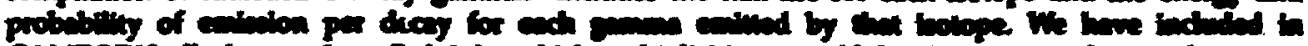

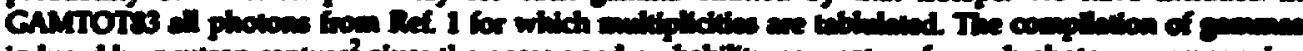

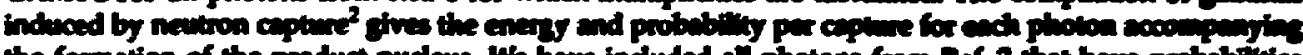

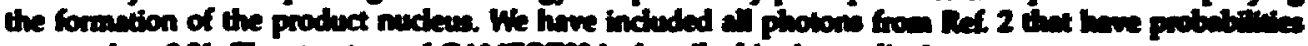
greater then 0.01. The structure of GAMTOTE3 is described in Append" $\mathrm{A}$.
\end{abstract}

\section{Using GAMIDEN} LUNL.

This section deals with the use of GAMIDEN per se; Appendix B details the use of GAMIDEN at

Most likely the user will want to enter the search constraints from his console so that he can alter them after seeing the program output. However, if there are many observed photon energies, he may want to enter photon energies from a user-created disk file. GAMIDEN allows the user to enter his dat from a console or from a disk file. The disk file contains the observed photon energies in format 10 F7.3. The last record of the data file should be blank. The program will request the name of the disk file, which must be less than or equal to eight characters.

The program prompts the user as follows:

\section{ENTER NAME OF SOURCE FILE IN A8, E.G., GAMTOT83}

This gives the user the option of using a data file other than GAMTOT83 if he wishes to do so. Of course, any other data file must be in the same format as GAMTOT83. (See Appendix A for a description of the required format.)

\section{ENTER DETECTOR SHIFTS LOWER, THEN UPPER IN 2F7.3}

Note that these uncertainties, although of the order of a few keV, are entered in $\mathrm{MeV}$. The user may use either a 2F7.3 format or a field-free, floating-point format and delimit the two variables with a comma.

\section{ENTER LOWER ENERGY LIMIT FOR GAMMAS IN F7.3}

This suppresses the search for energies less than the entered value. The unit of energy is MeV.

\section{ENTER LOWER AND UPPER LIMITS OF ISOTOPES IN 216}

These two numbers, entered as $1000 \mathrm{Z}+\mathrm{A}$, limit the range of isotopes searched.

ENTER LOWER BOUND of HALF-LIFE IN SEC. (F10.3)

This suppresses the search for unreasonably short half-lives. Enter zero if all isotopes are to be searched.

IF CAPTURE GAMMAS ONLY ENTER YES; OTHERWISE LF

A "YES" causes the program to ignore the previous entry and search only for gammas from Ref. 2 associated with neutron capture.

ENTER INPUT DEVICE FOR OBSERVED GAMMAS: 06 IF FILE; 59 IF TTY 


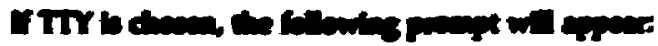

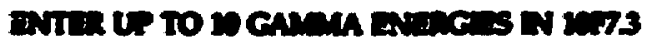

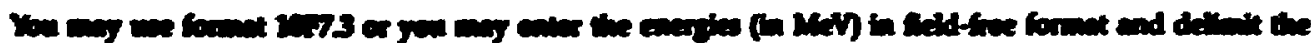

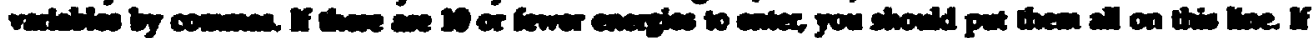

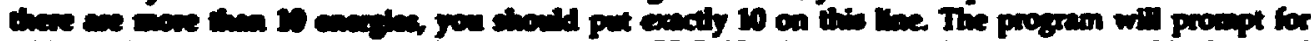

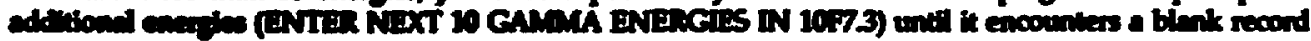

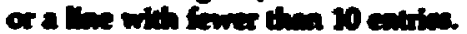

\section{ENTER LOWER DOUND OF MUTTIPLCTIIES IN F7.3}

This suppreaces the search for low-probability photons.

After output is completed the user is prompted for further problems:

\section{IF ANOTHER PRODLEM ENTER 1; IF NOT LF}

The program will request another set of observed photon energies.

\section{Example Input}

- Assume that observed photon energies are $0.058,0.826,1.173,1.332$, and $2.158 \mathrm{MeV}$.

- Consider all tabulated isotopes between ${ }_{20} \mathrm{Ca}^{40}$ and ${ }_{82} \mathrm{~Pb}^{207}$ with half-lives greater than $660 \mathrm{~s}$ (11 min).

- Consider all tabulated photons with energies greater than $0.80 \mathrm{MeV}$ and probabilities greater than $10 \%$.

- Accept as a match any photon that satisfies the criteria above and is within plus or minus $0.005 \mathrm{MeV}$ (5 keV) of an observed photon.

The user would enter these data from his console, as shown in the dialogue telow.

ENTER NAME OF SOURCE FILE IN A8, E.G., GAMTOT83

GAMTOT83

ENTER DETECTOR SHIFTS LOWER THEN UPPER IN 2 F7.3

$.005,005$

ENTER LOWER ENERGY LIMIT FOR GAMMAS IN F7.3

.80

ENTER LOWER AND UPPER LIMITS OF ISOTOPES IN 216 2004082207

ENTER LOWER BOUND OF HALF-LIFE IN SEC., (F10.3) 660.

IF CAPTURE GAMMAS ONLY ENTER YES; OTHERWISE LF

ENTER LOWER BOUND OF MULTIPLICITIES (F7.3)

$\underline{.1}$ 


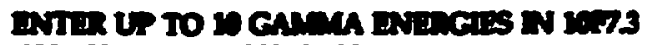

sen, $1.173,132,213$

If ANOTIER FOTIEM ENTER 1; IP NOT LF

\section{Sample Output}

HOUT, a disk file of revults, is generated when GAMIDEN is run. The reolts from the sample problem are liated and explained below.

1. The input variables are printed out (Fig 1).

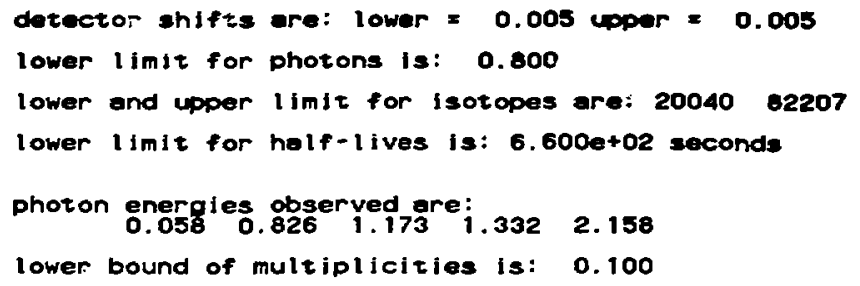

Figure 1.

2. The GAMTOT83 table is searched for isotopes that satisfy the input criteria. Each such isotope is printed out, accompanied by a list of other gamma emissions from the isotope that one might expect to see. The list below (Fig. 2), for the sample problem, gives the possible isotopes found in this way. The syr..vols used in the column labeled SOURCE are described in Appendix A.

nuclaus photon multiplifity half-lifo

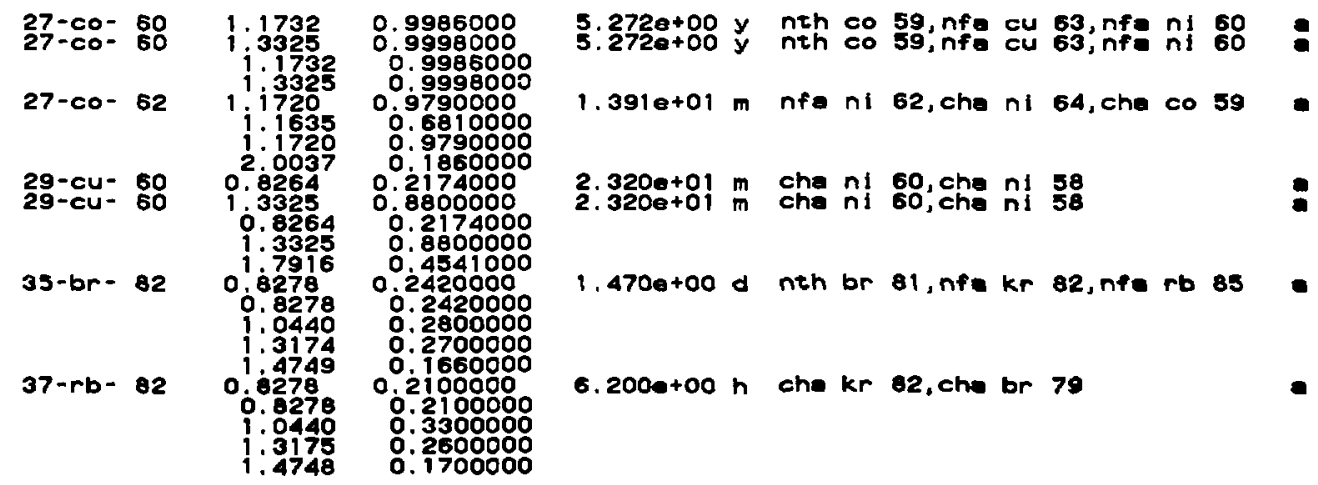

Figore 2. 




Figure 2. (Continued)

3. The elimination criterion (no unobserved higher-multiplicity photon) is applied, and those isotopes that survive this teat are listed (Fig. 3).

Of these candidates ${ }_{27} \mathrm{Co}^{\circ}$ is moat probable since it has two gaumms that meet the search criteria and both were observed. The other isotopes, for which only one of one or more pomible gammas were observed, are leas-probnble candidates. 


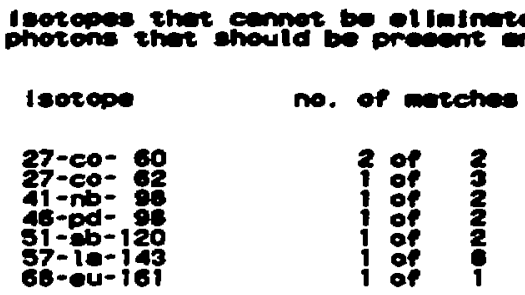

Figure 3.

4. The isotopes eliminated in the preceding step are listed (Fig. 4).

photons that should be observed and are not

$\begin{array}{lcc}\text { isotope } & \begin{array}{c}\text { least mult } \\ \text { observed }\end{array} & \begin{array}{c}\text { observed } \\ \text { photo }\end{array} \\ \text { 29-cu- } 60 & 0.2174 & 0.8264 \\ 35-b r-82 & 0.2420 & 0.8278 \\ 37-r b-82 & 0.2100 & 0.8278 \\ 45-r h-100 & 0.2060 & 0.8225 \\ 45-r h-106 & 0.1152 & 0.8245 \\ 47-a g-106 & 0.1543 & 0.8247 \\ 60-\mathrm{nd}-139 & 0.1025 & 0.8278 \\ 65-\mathrm{b}-160 & 0.1550 & 1.1780 \\ 67-\mathrm{ho}-166 & 0.1000 & 0.8306 \\ 69-\mathrm{tm}-168 & 0.1114 & 0.8211 \\ 79-\mathrm{au}-189 & 0.1000 & 0.8277 \\ 81-\mathrm{tl}-2.00 & 0.1100 & 0.8283\end{array}$

1.7916

0.4541

1.0440

0.2800

1.0440

0.3300

1.3175

0.2600

1.5534

0.2100

2.3761

0.3570

0.8040

1.0457

0.1152

o. 1344

1. 0458

o. 2972

0.9822

0.2618

0.8764

0.3000

0.9662

0.2550

0.8103

0.5970

0.8160

0. 4627

0.8126

0.9020

1.0715

0. 5900

0. 1000

0.2600

1. 2057

o. 3040

Figure 4. 


\section{References}


New York, 1979).

2. M. A Lone, R. A. Leavitt, and D. A. Harrion, Atomic Date and Nuclear Data Tables, 26,511 (1931).

HJK/mc 


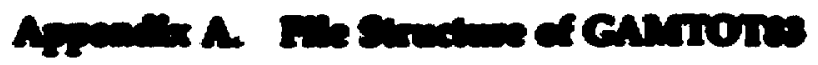

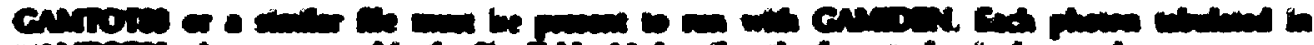

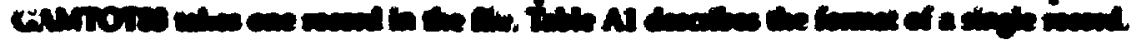

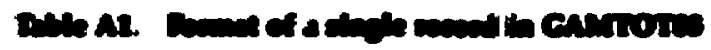

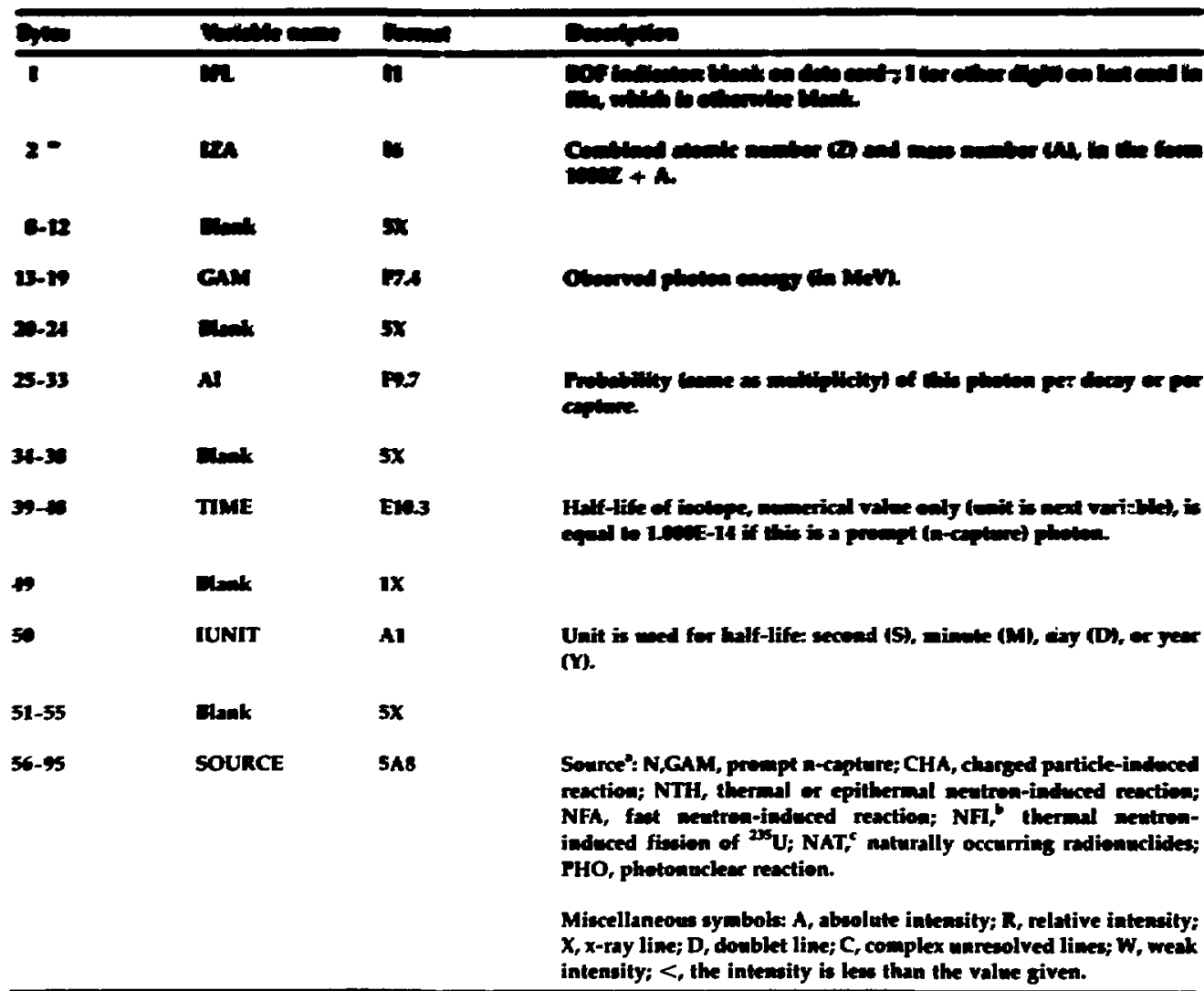

'For all symbols except NFI and NAT the nuclide designator that follows refers to the target nuclide.

"For NFI the number following the symbol refers to the cumulative yield of the fiesion product.

'For NAT the number following the symbol is the percentage of natural isotopic abundance. 


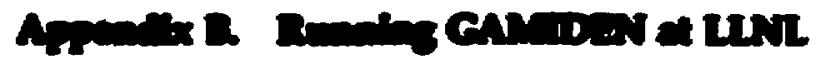

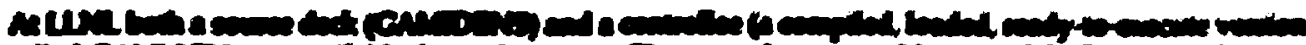

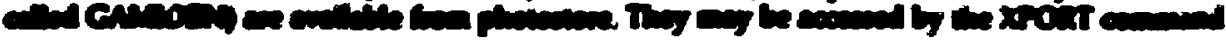

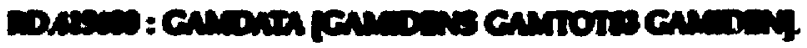

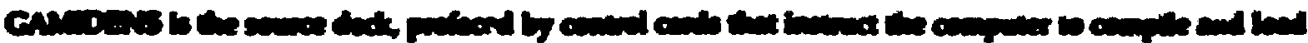

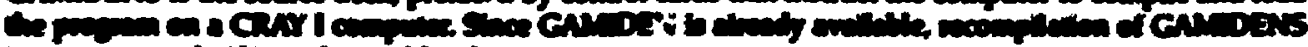



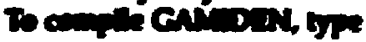

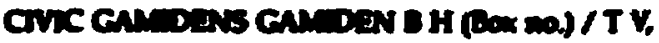



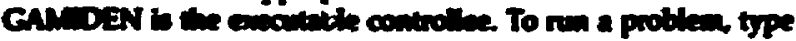

\section{CAMDEN / T V.}

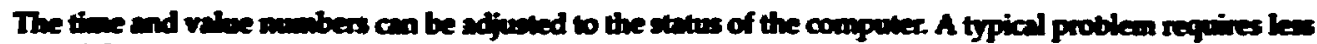
then 0.75 min.

Pleave note that diat file GAMTOTr3 must be present. 